\title{
Planet formation in action: resolved gas and dust images of a transitional disk and its cavity
}

\author{
Nienke van der Marel ${ }^{1}$, Ewine F. van Dishoeck ${ }^{1,2}$, Simon Bruderer ${ }^{2}$, \\ Til Birnstiel ${ }^{3}$, Paola Pinilla ${ }^{4}$, Cornelis P. Dullemond ${ }^{4}$, Tim A. van \\ Kempen $^{1,5}$, Markus Schmalzl ${ }^{1}$, Joanna M. Brown ${ }^{3}$, Gregory J. \\ Herczeg $^{6}$, Geoffrey S. Mathews ${ }^{1}$ and Vincent Geers ${ }^{7}$ \\ ${ }^{1}$ Leiden Observatory, Leiden University, P.O. Box 9513, 2300 RA Leiden, The Netherlands, \\ ${ }^{2}$ Max-Planck-Institut für Extraterrestrische Physik Giessenbachstrasse 1, 85748 Garching, \\ Germany, \\ ${ }^{3}$ Harvard-Smithsonian Center for Astrophysics, 60 Garden Street, Cambridge, MA 02138, \\ USA \\ ${ }^{4}$ Heidelberg University, Center for Astronomy, Institute for Theoretical Astrophysics, Albert \\ Ueberle Str. 2, 69120, Heidelberg, Germany, \\ ${ }^{5}$ Joint ALMA Offices, Av. Alonso de Cordova, Santiago, Chile \\ ${ }^{6}$ Kavli Institute for Astronomy and Astrophysics, Peking University, Yi He Yuan Lu 5, Hai \\ Dian Qu, 100871 Beijing, PR China \\ ${ }^{7}$ Dublin Institute for Advanced Studies, 31 Fitzwilliam Place, Dublin 2, Ireland
}

\begin{abstract}
Planet formation and clearing of protoplanetary disks is one of the long standing problems in disk evolution theory. The best test of clearing scenarios is observing systems that are most likely to be actively forming planets: the transitional disks with large inner dust cavities. We present the first results of our ALMA (Atacama Large Millimeter/submillimeter Array) Cycle 0 program using Band 9, imaging the Herbig Ae star Oph IRS 48 in CO 6-5 and the submillimeter continuum in the extended configuration. The resulting $\sim 0.2^{\prime \prime}$ spatial resolution completely resolves the cavity of this disk in the gas and the dust. The gas cavity of IRS 48 is half as large as the dust cavity, ruling out grain growth and photoevaporation as the primary cause of the truncation. On the other hand, the continuum emission reveals an unexpected large azimuthal asymmetry and steep edges in the dust distribution along the ring, suggestive of dust trapping. We will discuss the implications of the combined gas and dust distribution for planet formation at a very early stage. This is one of the first transition disks with spatially resolved gas inside the cavity, demonstrating the superb capabilities of the Band 9 receivers.
\end{abstract}

Keywords. planetary systems: formation,planetary systems: protoplanetary disks

\section{Introduction}

Transitional disks are protoplanetary disks around young stars with a hole or gap in their small dust grain distribution. These disks possibly form the evolutionary link between optically thick protoplanetary disks around $\mathrm{T}$ Tauri stars and optically thin debris disks around older systems (Williams \& Cieza 2011). There are three different mechanisms in this phase of disk evolution that could cause the appearance of such a dust hole (e.g. Najita et al. 2007): (i) increased grain growth in the central region of the disk that lowers the emitting surface area below the detectable limit; (ii) photoevaporation by the radiation from the star; (iii) the clearing by either a stellar or (proto)planetary 

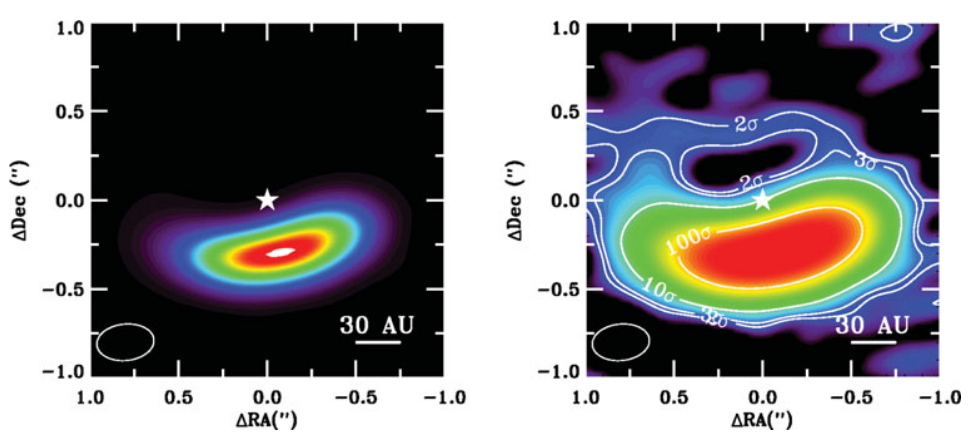

Figure 1. The inclined disk around IRS 48 in dust as observed with ALMA Band 9 observations. The ALMA beam during the observations is indicated with a ellipse. The figure shows the 685 $\mathrm{GHz}$ continuum emission in linear (left) and logarithmic color scale (right), with white contours at $2,3,10$ and $100 \sigma(\sigma=0.82 \mathrm{mJy} /$ beam $)$.

companion. It is therefore not possible to assign the origin of the hole by only looking at the dust distribution. However, the gas distribution will depend on the mechanism: in the case of grain growth, the gas density is not affected at all and there will still be gas present inside the dust hole; in the case of photoevaporation, the gas and dust are cleared simultaneously from the inside out; in the case of a companion the dust hole is expected to be larger than the gas hole and an inner (dust) disk may still be present. The key to understanding the origin of the hole in a transitional disk is therefore resolving both the gas and dust distribution at scales of $<50 \mathrm{AU}$, which ALMA finally allows us to do.

We present ALMA Cycle 0 results of one of these transitional disks, Oph IRS 48 (van der Marel et al. 2013). This Herbig Ae star, located in the star forming region Ophiuchus, was already known as a peculiar disk: VLT-VISIR resolved mid infrared imaging shows a large dust ring at $18.7 \mu \mathrm{m}$ with a radius of $\sim 55 \mathrm{AU}$ (Geers et al. 2007), whereas VLTCRIRES near infrared observations of the rovibrational CO $v=1-0$ line reveal a hot gas ring at $30 \mathrm{AU}$ radius (Brown et al. 2012a). However, both observations only address the hot dust and gas in the inner disk that is directly heated by the star: submillimeter observations are required for the bulk cold disk material. At the distance of Ophiuchus $(120 \mathrm{pc})$, the spatial resolution of ALMA $\left(\sim 0.2^{\prime \prime}\right)$ provides a beam radius of $\sim 14 \mathrm{AU}$, sufficient to distinguish between these two reported hole sizes and resolve the disk in both the gas and the dust. The ALMA observations were taken in July 2012, using the Band 9 instrument $(\sim 690 \mathrm{GHz})$ in the Extended Configuration, tracing both the ${ }^{12} \mathrm{CO}$ 6-5 line and the $685 \mathrm{GHz}$ dust continuum.

\section{Results}

The ${ }^{12} \mathrm{CO}$ data show a full Keplerian gas disk at $50^{\circ}$ inclination, except for a strong absorption around $v_{\mathrm{LSR}}=3.5 \mathrm{~km} \mathrm{~s}^{-1}$ due to foreground clouds. Otherwise, the spectral profile is symmetric around the source velocity $\left(4.5 \mathrm{~km} \mathrm{~s}^{-1}\right)$ and the highest velocity channels are consistent with a gas hole with a radius of $20 \mathrm{AU}$ (van der Marel et al. 2013, Bruderer et al. 2013). On the other hand, the dust continuum emission that was expected to be ring-shaped, similar to the mid infrared emission, reveals a highly asymmetric structure on the southern side of the disk (see Figure 1). This crescent feature has a peak intensity of $390 \sigma$, with $\sigma=0.8 \mathrm{mJy} / \mathrm{beam}$, with no significant detectable emission in the northern part of the disk, indicating a contrast of a factor $>130$. 
The crescent could be fitted with an arc with an azimuthal extent of $\sim 100^{\circ}$ and a radial extent between 45 and 80 AU from the star. Combining the total $690 \mathrm{GHz}$ flux with SMA fluxes at 345 and $230 \mathrm{GHz}$ (Brown et al. 2012b), the spectral slope $\alpha$ $\left(F_{\nu} \sim \nu^{\alpha}\right)$ is $2.7 \pm 0.25$, consistent with dust growth up to millimeter sizes. Assuming a dust temperature of $60 \mathrm{~K}$, the total dust mass of the millimeter grains is derived as $\sim 9$ Earth masses. Considering the high concentration of millimeter dust particles in one region of the disk, it is expected that the dust grains in this region will grow up to a kilometer in size (planetesimal sizes) before being scattered around the ring, so the crescent can be considered as a Kuiper Belt Object factory (van der Marel et al. 2013). Comparing the crescent of millimeter dust with the VISIR image at $18.7 \mu \mathrm{m}$ (tracing the hot micron-sized dust particles), there is not only a separation between the gas and the millimeter dust, but also between the small and large dust particles (Figure 1).

\section{Implications: dust trapping}

The size of the gas hole in combination with the radial extent of the dust continuum implies that a companion is responsible for the hole in this transitional disk. This can be understood in the framework of dust trapping. In a 'normal' protoplanetary disk without holes or gaps, dust evolution is governed by a balance between dust coagulation and fragmentation (by collisions) and radial inward drift (Weidenschilling 1977).

Radial drift is caused by the interaction with the gas: the gas in a Keplerian disk moves at sub-Keplerian speeds, since it feels the force of its own pressure gradient which is usually pointing inwards. Small dust particles are coupled to the gas and move along the same orbits, while larger dust grains decouple from the gas and approach Keplerian velocities. Therefore the larger dust particles feel a constant head wind, which causes the particle to lose angular momentum and drift inwards. As a consequence, large dust particles will always move towards regions of higher pressure. One of the main problems in dust evolution modeling is that dust particles in the outer disk can not grow beyond millimeter sizes because of their rapid inward drift ('radial drift barrier').

One of the proposed solutions for the radial drift barrier is dust trapping in local pressure maxima in the disk. Pinilla et al. (2012) used a combination of FARGO hydrodynamic models in combination with dust evolution modeling (Birnstiel et al. 2010) to show that when a planet is placed in orbit in a gas disk, it will quickly clear its path and create an overdensity (radial pressure bump) at the edge of the gap, where the large dust particles accumulate within $10^{5}$ years and remain trapped, allowing further dust growth. The created dust hole will always be larger than the gas hole, with their sizes depending on the viscosity and the planet mass. The radial separation between gas and dust in IRS 48 indicates that the companion has to be massive, $>10 M_{\text {Jup }}$ (van der Marel et al. 2013). It was demonstrated that for such a massive companion within a disk of moderate viscosity the pressure bump becomes Rossby unstable and quickly forms a long-lived vortex in the gas. Such a vortex (a small overdensity in the gas) can trap the dust in both the radial and azimuthal direction, where the trapping efficiency depends on the size of the dust particle and the viscosity (Birnstiel et al. 2013). An overdensity of a factor $2-3$ in the gas can lead to more than a factor of 100 overdensity in the millimeter dust. The high asymmetry in the dust crescent in IRS 48 could thus be reproduced using this scenario (van der Marel et al. 2013).

We propose the dust trapping scenario with a $10 M_{\mathrm{Jup}}$ companion at $20 \mathrm{AU}$ as an explanation for the separation between the millimeter-sized dust, gas and micron-sized dust in IRS 48. A dust trap located at $60 \mathrm{AU}$ distance from the star has an orbital period of 330 years, so the orbital shift will be measurable with ALMA within 20 years. This dust 
trap, acting as Kuiper Belt Object factory, is analogous to a planet factory at smaller radii around other stars. The observational evidence for the dust trapping mechanism may provide a new view on planet formation and disk evolution models. The question remains whether IRS 48 is a special case, or if dust trapping is a common phenomenon in transition disks. ALMA Cycle 0 observations of the transitional disk HD142527 also show a high dust asymmetry without large azimuthal variations in the gas (Casassus et al. 2013). ALMA will be able to provide answers for other transition disks, providing even higher spatial resolution in the coming years, allowing both the gas and dust to be resolved.

\section{References}

Birnstiel, T., Dullemond, C. P., \& Brauer, F. 2010, A\&SA, 513, A79

Birnstiel, T., Dullemond, C. P., \& Pinilla, P. 2013, A\&A, 550, L8

Brown, J. M., Herczeg, G. J., Pontoppidan, K. M., \& van Dishoeck, E. F. 2012, ApJ, 744, 116

Brown, J. M., Rosenfeld, K. A., Andrews, S. M., Wilner, D. J., \& van Dishoeck, E. F. 2012, ApJ, 758, 30

Bruderer, S., van der Marel, N., van Dischoeck, E. F., \& van Kempen, T. A., 2013, A\&\&A, submitted

Casassus, S., van der Plas, G., M, S. P., Dent, W. R. F., Fomalont, E., Hagelberg, J., et al. 2013, Nature, 493, 191

Geers, V. C., Pontoppidan, K. M., van Dishoeck, E. F., Dullemond, C. P., Augereau, J.-C., et al. 2007, A\&A, 469, L35

Najita, J. R., Strom, S. E., \& Muzerolle, J., 2007, MNRAS, 378, 369

Pinilla, P., Benisty, M., \& Birnstiel, T., 2012, A\& $A$, 545, A81

van der Marel, N., van Dishoeck, E. F., Bruderer, S., Birnstiel, T., Pinilla, P., et al. 2013, Science, 340,1199

Williams, J. \& Cieza, L. 2011, ARAA, 49, 67

Weidenschilling, S. J. 1977, MNRAS, 180, 57

\section{Discussion}

BARSONY: I had e-mailed Nienke an image of IRS 48 at 12.5 micron that is very similar to the 18.7 micron image. I just wanted to note that systems like this may be common or not, but in terms of detecting the small particles by mid infrared continuum, that is going to be incredibly difficult. We observed a 100 sources in $\rho$ Oph with Keck with a mid infrared instrument and this was the only source that showed this kind of structure. Since it is an A0 star it has enough UV to excite the small PAHs and the very small grains so you can see it reradiating in the mid infrared, but for lower mass systems it would be impossible to find where the small dust particles are.

Hales Gebrim: You show that the Rossby instability can reproduce the submillimeter data well. Have you tried plugging the same model in radiative transfer code to see if it can also reproduce the scattered light or mid infrared images?

VAN DER MAREL: No, we haven't done that yet, but it's something we would like to try.

DonG: Is it possible to confirm the presence of the planet?

VAN DER MAREL: It would be difficult, because this disk is in a region of very high optical extinction, so optical direct imaging will be impossible. We hope there will be some result from a NACO program with Sparse Aperture Masking, looking for a companion in the near infrared. But this is still work in progress. 